\title{
ON THE PROBLEM OF RETRACTING BALLS ONTO THEIR BOUNDARY
}

\author{
KAZIMIERZ GOEBEL
}

Received 13 November 2001

We provide some new estimates of the smallest possible Lipschitz constant for retractions of the unit ball $B$ onto the unit sphere $S$ in infinite-dimensional Banach spaces.

\section{Introduction}

Let $(X,\|\cdot\|)$ be an infinite-dimensional Banach space with the unit ball $B$ and the unit sphere $S$. Since the works of Nowak [11], Benyamini and Sternfeld [1], and Lin and Sternfeld [10], it is known that $S$ is a Lipschitzian retract of $B$. It means that there exists a mapping (retraction) $R: B \rightarrow S$ satisfying $R x=x$ for all $x \in S$ and also being Lipschitzian. If $R$ satisfies the Lipschitz condition with constant $k(R \in \mathscr{L}(k))$,

$$
\|R x-R y\| \leq k\|x-y\|
$$

then $k$ cannot be too small. There is an interesting question: what is the infimum of all $k$ admitting existence of a retraction $R: B \rightarrow S$ of class $\mathscr{L}(k)$ ? For a given space $X$, we denote this constant by $k_{0}(X)$. Now the exact value of this constant $k_{0}(X)$ is not known for any space $X$. The basic evaluations of $k_{0}(X)$ can be found in [7] by Kirk and Goebel. They partially came from Franchetti [5], Goebel and Komorowski [8], and Komorowski and Wośko [9]. Some recent evaluations can be found in Bolibok and Goebel [4] and in Bolibok [2, 3]. Here is a list of some known facts.

(A) Evaluation from below: $k_{0}(X) \geq 3$ for all spaces, $k_{0}(X)>3$ for all uniformly convex spaces, $k_{0}(H) \geq 4.5 \ldots$ for Hilbert space, and $k_{0}\left(l^{1}\right)>4$.

(B) Evaluation from above: there is a universal constant $k_{0}$ such that for all spaces $X, k_{0}(X) \leq k_{0}$ but good evaluations of $k_{0}$ are unknown, 


$$
k_{0}\left(L^{1}(0,1)\right) \leq 9.43 \ldots, \quad k_{0}(C[0,1]) \leq 4(1+\sqrt{2})^{2}=23.31 \ldots,
$$

but

$$
k_{0}\left(C_{0}[0,1]\right) \leq 15.82 \ldots
$$

where $C_{0}[0,1]$ is the subspace of $C[0,1]$ consisting of functions vanishing at zero,

$$
k_{0}\left(c_{0}\right)<35.18 \ldots, \quad k_{0}\left(l^{1}\right)<31.64 \ldots, \quad k_{0}(H)<31.45 \ldots .
$$

Remark 1.1. There are no universal methods for finding such evaluations. Most of them have been obtained via tricky individual constructions. Our aim is to present some samples which are modifications of known results (see, e.g., [6]). We hope they may attract the reader's attention to the subject.

First recall the so-called minimal displacement problem. Let $T: B \rightarrow B$ be of class $\mathscr{L}(k)$. It may happen that $T$ is fixed point free and even more $d_{T}=$ $\inf \{\|x-T x\|: x \in B\}$ is positive.

For any space $X$, we define the function

$$
\psi(k)=\sup _{T \in \mathscr{L}(k)} \inf _{x \in B}\|x-T x\|=\sup _{T \in \mathscr{L}(k)} d_{T}
$$

Since, in view of Banach fixed-point theorem, for any $\varepsilon>0$, the equation

$$
x=\frac{1}{k+\varepsilon} T x
$$

has a solution, we have

$$
d_{T} \leq\|x-T x\|=\left\|\frac{1}{k+\varepsilon} T x-T x\right\|=\left(1-\frac{1}{k+\varepsilon}\right)\|T x\| \leq\left(1-\frac{1}{k+\varepsilon}\right)
$$

implying

$$
\psi(k) \leq 1-\frac{1}{k}
$$

For some spaces, this estimate is the best possible. For example, this is the case for $C[-1,1]$ (or any $C[a, b]$ ). 
Let

$$
\alpha(t)= \begin{cases}-1 & \text { for } t \leq-1 \\ t & \text { for }-1 \leq t \leq 1 \\ 1 & \text { for } t \geq 1\end{cases}
$$

The function $\alpha$ generates the retraction $Q: C[-1,1] \rightarrow B$ of class $\mathscr{L}(1)$ defined by

$$
(Q f)(t)=\alpha(f(t))
$$

We leave to the reader the justification of the fact that the mapping $T: B \rightarrow B$, defined by

$$
(T x)(t)=\alpha(k(x(t)+2 t))
$$

is of class $\mathscr{L}(k)$ with $d_{T}=1-1 / k$. Thus for $X=C[0,1], \psi(k)=1-1 / k$.

The same holds for many other square spaces like $c_{0}, c, l^{\infty}, C^{n}[a, b]$, and all the subspaces of $C[a, b]$ of finite codimension. We call such spaces extremal. Uniformly convex spaces, Hilbert space, but also $l^{1}$, are not extremal.

Since the discussed function depends on the geometry of the space $X$, if necessary, we will indicate this writing $\psi(k)=\psi_{X}(k)$. List some basic properties of $\psi$ valid for all spaces $X$ :

(a) $\psi(k)$ is nondecreasing,

(b) $\lim _{k \rightarrow \infty} \psi(k)=1$,

(c) $\psi(1-\alpha+\alpha k) \geq \alpha \psi(k)$ for all $0 \leq \alpha \leq 1$,

(d) $\psi(k) /(k-1)$ is nonincreasing,

(e) $k \psi(k) /(k-1)$ is nondecreasing,

(f) $\psi^{\prime}(1)=\lim _{k \rightarrow 1^{+}} \psi(k) /(k-1)$ exists and $\psi^{\prime}(1)>0$,

(g) $\psi(k) \geq \psi^{\prime}(1)(1-1 / k)$,

(h) $X$ is extremal if and only if $\psi^{\prime}(1)=1$.

Property (a) is trivial, (b) is an exercise for the reader, (c) follows from the simple observation that if $T \in \mathscr{L}(k)$ and $\alpha \in[0,1]$, then $T_{\alpha}=(1-\alpha) I+\alpha T \in$ $\mathscr{L}(1-\alpha+\alpha k)$ with $\left\|x-T_{\alpha} x\right\|=\alpha\|x-T x\|$ for all $x \in B$. Next, (d) is a reformulation of (c). Since property (e) plays a role in our further considerations, we present its proof. Then (f), (g), and (h) easily follow.

Take any $A>k$. Let $T \in \mathscr{L}(k)$. Fix $x \in B$ and consider the equation

$$
y=\left(1-\frac{1}{A}\right) x+\frac{1}{A} T y .
$$


104 On the problem of retracting balls onto their boundary

In view of Banach contraction principle, it has exactly one solution $y$ depending on $x$. Put $y=F x$. We have the implicit formula

$$
F x=\left(1-\frac{1}{A}\right) x+\frac{1}{A} T F x
$$

from which we get $F \in \mathscr{L}((A-1) /(A-k))$ and $T F \in \mathscr{L}(k((A-1) /(A-k)))$. Also if $\|x-T x\| \geq d>0$ for all $x \in B$, then

$$
\|x-T F x\|=\frac{A}{A-1}\|F x-T F x\| \geq \frac{A d}{A-1} .
$$

Consequently,

$$
\psi\left(\frac{k(A-1)}{A-k}\right) \geq \frac{A}{A-1} \psi(k) .
$$

Denoting $k(A-1) /(A-k)$ by $l$ (observe that $l>k$ ), we obtain

$$
\frac{l \psi(l)}{l-1} \geq \frac{k \psi(k)}{k-1}
$$

which ends the proof.

Remark 1.2. It is naturally expected that there are some relations between the function $\psi_{X}(k)$ and the constants $\psi^{\prime}(1)$ and $k_{0}(X)$.

\section{General case}

Now we pass to a certain scheme for construction of Lipschitzian retraction of $B$ onto $S$. Suppose we have a mapping $T \in \mathscr{L}(k), T: B \rightarrow X$ (not necessarily $T: B \rightarrow B$ ) such that $d_{T}>0$. Assume additionally that for all $x \in S, T x=0$. It will be shown that such mappings do exist. Each one generates the retraction $R: B \rightarrow S$ defined by

$$
R x=\frac{x-T x}{\|x-T x\|}=P\left(\frac{x-T x}{d_{T}}\right)
$$

where $P: X \rightarrow B$ is the radial projection

$$
P x= \begin{cases}x & \text { for }\|x\| \leq 1 \\ \frac{x}{\|x\|} & \text { for }\|x\|>1\end{cases}
$$


Since $P \in \mathscr{L}(2)$, we have $R \in \mathscr{L}\left(2\left((k+1) / d_{T}\right)\right)$. Consequently,

$$
k_{0}(X) \leq 2 \frac{k+1}{d_{T}}
$$

We can try to optimize this evaluation selecting various mappings $T$.

Begin with a mapping $T_{0}: B \rightarrow B$ of class $\mathscr{L}(k)$ with $d_{T_{0}}=d>0$. Extend $T_{0}$ to $T_{1}: 2 B \rightarrow B$ by setting

$$
T_{1} x= \begin{cases}T_{0} x & \text { for }\|x\| \leq 1, \\ (2-\|x\|) T_{0} P x & \text { for } 1<\|x\| \leq 2\end{cases}
$$

Thus $T_{1} \in \mathscr{L}(2 k+1)$ and for all $x \in 2 S$, we have $T x=0$. For $x \in B$, we have

$$
\left\|x-T_{1} x\right\|=\left\|x-T_{0} x\right\| \geq d .
$$

For $x \in 2 B \backslash B$, we have

$$
\begin{aligned}
\left\|x-T_{1} x\right\| & \geq\left\|x-T_{0} P x\right\|-\left\|T_{0} P x-(2-\|x\|) T_{0} P x\right\| \\
& \geq\left\|P x-T_{0} P x\right\|-\|x-P x\|-(\|x\|-1)\left\|T_{0} P X\right\| \\
& \geq d-2(\|x\|-1) .
\end{aligned}
$$

On the other hand,

$$
\left\|x-T_{1} x\right\| \geq\|x\|-\left\|T_{1} x\right\|=\|x\|-(2-\|x\|)\left\|T_{0} P x\right\| \geq 2(\|x\|-1) .
$$

Consequently,

$$
d_{T_{1}} \geq \min _{t \in[1,2]}\{d-2(t-1), 2(t-1)\}=\frac{d}{2}
$$

Finally, putting

$$
T x=\frac{1}{2} T_{1}(2 x)
$$

we obtain a mapping $T: B \rightarrow B$ of class $\mathscr{L}(2 k+1)$ with $d_{T} \geq d / 4$ such that $T(S)=$ $\{0\}$. 
Using (2.1), we construct a retraction $R: B \rightarrow S$ of class $\mathscr{L}(16((k+1) / d))$. Observing that the initial mapping can be selected so that $d$ is close to $\psi(k)$, we come to the conclusion that for any space $X$,

$$
k_{0}(X) \leq 16 \frac{k+1}{\psi(k)}
$$

for all $k>1$. Using the above mentioned properties (e), (f), and (g) of $\psi(k)$, we obtain

$$
k_{0}(X) \psi^{\prime}(1) \leq 16 \min _{k>1} \frac{k(k+1)}{k-1}=16(1+\sqrt{2})^{2}=93.25 \ldots
$$

The above evaluation ties two unknown values $k_{0}(X)$ and $\psi^{\prime}(1)$. Since all the estimates used in deriving it were very rough, the above evaluation is, probably, very imprecise. For example, it is known (see [4]) that in the case of extremal spaces where $\psi^{\prime}(1)=1$, we have $k_{0}(X) \leq 37.74 \ldots$. An interesting and a little stronger evaluation can be derived from $(2.10)$ by rewriting it in the form

$$
k_{0}(X) \frac{k \psi(k)}{k-1} \leq 16 \frac{k(k+1)}{k-1}
$$

and observing that the left-hand side increases with $k$ but the right-hand side of the above inequality decreases until it takes the minimal value at $k=1+\sqrt{2}$. At this point, we get

$$
k_{0}(X) \psi(1+\sqrt{2}) \leq 16(2+\sqrt{2})=54.62 \ldots
$$

Since, besides extremal spaces, good evaluations of $\psi(k)$ from below are practically unknown, the estimates of Section 2 have to be treated with reserve. In next sections, we show some better estimates in particular spaces.

\section{The case of $C[0,1]$}

Let $X=C[0,1]$. As we observed in the introduction, besides the radial projection $P: C[0,1] \rightarrow B$, there exists also the projection $Q: C[0,1] \rightarrow B$ of class $\mathscr{L}(1)$ defined by $Q f=\alpha \circ f$. Consequently, for any $r>0$ there exists the retraction $Q_{r}: C[0,1] \rightarrow B_{r}=r B$ of class $\mathscr{L}(1)$ defined by

$$
\left(Q_{r} f\right)(t)=\alpha_{r}(f(t))=r \alpha\left(\frac{f(t)}{r}\right)
$$

It is not difficult to check that for any $r_{1}>0, r_{2}>0$, and $f, g \in C[0,1]$,

$$
\left\|Q_{r_{1}} f-Q_{r_{2}} g\right\| \leq \max \left\{\|f-g\|,\left|r_{1}-r_{2}\right|\right\}
$$


As we have already shown, $C[0,1]$ is extremal and for any $k>1$ there exists a mapping $T: B \rightarrow B$ of class $\mathscr{L}(k)$ such that $d_{T}=1-1 / k$. Consider the ball of radius $2\left(B_{2}=2 B\right)$ and extend $T$ to the mapping $T_{1}: 2 B \rightarrow B$ by putting

$$
T_{1} f= \begin{cases}T f & \text { for }\|f\| \geq 1, \\ T(Q f) & \text { for } 1<\|f\| \leq 2-\frac{1}{k}, \\ Q_{k(2-\|f\|)}(T(Q f)) & \text { for } 2-\frac{1}{k} \leq\|f\| \leq 2 .\end{cases}
$$

Again $T_{1} \in \mathscr{L}(k)$, and for all $f \in 2 S$ we have $T_{1} f=0$. Moreover, it can be observed that $\left\|f-T_{1} f\right\| \geq 1-1 / k$. Now denoting

$$
\widetilde{T} f=\frac{1}{2} T_{1}(2 f)
$$

we get a mapping $\widetilde{T}: B \rightarrow B$ with $d_{T}=(1 / 2)(1-1 / k)$ and $\tilde{T}(S)=0$. Finally, we can generate a retraction $R: B \rightarrow B$ by putting

$$
R f=\frac{f-\widetilde{T} f}{\|f-\widetilde{T} f\|}=P\left(\frac{f-\widetilde{T} f}{(1 / 2)(1-1 / k)}\right) .
$$

As before, we observe that $R \in \mathscr{L}(4((k+1) k /(k-1)))$ and therefore,

$$
k_{0}(C[0,1]) \leq 4 \min _{k>1} \frac{(k+1) k}{k-1}=4(1+\sqrt{2})^{2}=23.31 \ldots
$$

Probably, this estimate is far from being sharp but, according to our knowledge, it is the best known.

\section{The case of Hilbert space}

Let $H$ be a Hilbert space. In this case, the radial projection $P$ of $H$ onto the unit ball $B$ is of class $\mathscr{L}(1)$ and, moreover, for any $x \in H \backslash B$ and any $y \in B$, we have $(x-P x, P x-y) \geq 0$ implying $\|x-y\| \geq\|P x-y\|$. The same holds, for any $r>0$, for radial projection $P_{r}$ onto the ball $B_{r}=r B$.

Let $T_{0}: B \rightarrow B$ be a mapping of class $\mathscr{L}(k)$ with $d_{T_{0}}=d>0$. Standard Hilbert space calculations show that if $\|x\| \geq \sqrt{1-d^{2}}$, then $\left(T_{0} x-x, x\right) \leq 0$. This allow us to modify the mapping $T_{0}$ by defining $T_{1}: B \rightarrow B$ in the following way:

$$
T_{1} x= \begin{cases}T_{0} x & \text { for }\|x\| \leq \sqrt{1-d^{2}} \\ T_{0} P_{\sqrt{1-d^{2}}} x & \text { for } \sqrt{1-d^{2}} \leq\|x\| \leq 1\end{cases}
$$


The modified mapping $T_{1}$ is also of class $\mathscr{L}(k)$ with $d_{T_{1}} \geq d_{T_{0}}=d$. Moreover, for any $x \in S$,

$$
\left\|x-T_{1} x\right\| \geq \sqrt{2\left(1-\sqrt{1-d^{2}}\right)}>d
$$

Again, some Hilbert space geometry calculations show that for any $x \in S, \mu \in$ $[0,1]$ and any $\lambda>1$, we have

$$
\left\|\mu T_{1} x-\lambda x\right\| \geq d
$$

First, we construct an extension of $T_{1}$ by defining the domain $D=\{x \in H$ : $\left.\|x\| \leq 1+1 / k-(1 / k)\left\|T_{1} P x\right\|\right\}$ and the mapping $T_{2}: D \rightarrow B$

$$
T_{2} x= \begin{cases}T_{1} x & \text { for } x \in B, \\ T_{1} P x & \text { for } x \in D \backslash B\end{cases}
$$

Obviously, $T_{2} \in \mathscr{L}(k)$ and $d_{T_{2}}=d$. In the next step, observe that the mapping $T_{3}: D \cup(1+1 / k) S \rightarrow B$, defined by

$$
T_{3} x= \begin{cases}T_{2} x & \text { for } x \in D \\ 0 & \text { for } x \in\left(1+\frac{1}{k}\right) S\end{cases}
$$

is also of class $\mathscr{L}(k)$ with $d_{T_{3}}=d$.

Finally, the mapping $T_{3}$ can be extended to a mapping $T_{4}:(1+1 / k) B \rightarrow B$, again of class $\mathscr{L}(k)$ via the use of the well-known Kirszbraun's theorem. However, since for each $x \in S$, such extension has to map the segment joining ( $1+$ $\left.1 / k-(1 / k)\left\|T_{1} P x\right\|\right) x$ and $(1+1 / k) x$ of length $(1 / k)\left\|T_{1} P x\right\|$ onto an arc joining $T_{1} P x$, and 0 of length exceeding $\left\|T_{1} P x\right\|$. This extension can be done in exactly one way

$$
T_{4} x= \begin{cases}T_{2} x & \text { for } x \in D, \\ k\left(1+\frac{1}{k}-\|x\|\right) \frac{T_{1} P x}{\left\|T_{1} P x\right\|} & \text { for } x \in\left(1+\frac{1}{k}\right) B \backslash D .\end{cases}
$$

For points with $T_{1} P x=0$, obviously $T_{4} x=0$. In view of (4.3) it is clear that $d_{T_{4}}=d$. 
Coming back to the unit ball, we obtain the mapping $T: B \rightarrow B$

$$
T x=\frac{k}{k+1} T_{4}\left(\frac{k+1}{k} x\right)
$$

of class $\mathscr{L}(k)$ such that $T(S)=\{0\}$ and $d_{T}=(k /(k+1)) d$.

Our standard construction

$$
R x=\frac{x-T x}{\|x-T x\|}=P\left(\frac{x-T x}{d_{T}}\right)=P\left(\frac{k+1}{k} \frac{x-T x}{d}\right),
$$

in view of the fact that $P \in \mathscr{L}(1)$ and $T_{0}$ can be chosen so that $d$ is close to $\psi_{H}(k)$, leads to the estimate

$$
k_{0}(H) \leq \frac{(k+1)^{2}}{k \psi_{H}(k)} \quad \forall k>1
$$

Consequently, in view of property $(\mathrm{g})$ of $\psi$,

$$
k_{0}(H) \psi_{H}^{\prime}(1) \leq \min _{k>1} \frac{(k+1)^{2}}{k-1}=8 .
$$

The minimum is taken at $k=3$. Repeating the trick from Section 3 we can rewrite (4.9) in the following way:

$$
k_{0}(H) \frac{k \psi_{H}(k)}{k-1} \leq \frac{(k+1)^{2}}{k-1}
$$

and taking the optimal value of $k, k=3$, we get

$$
k_{0}(H) \psi_{H}(3) \leq \frac{16}{3}
$$

\section{Final remarks}

All the above constructions and evaluations seem to be very imprecise. This comes from the fact that the evaluation from below of the function $\psi$, indicated by property $(\mathrm{g})$, is not sharp for nonextremal spaces. It is known that $\lim _{k \rightarrow \infty} \psi(k)=1$. The problem of finding $k_{0}(X)$ for at least one space $X$ remains open.

\section{Acknowledgment}

This work was supported by the State Committee for Scientific Research of Poland (KBN) grant 2P03A02915. 
110 On the problem of retracting balls onto their boundary

\section{References}

[1] Y. Benyamini and Y. Sternfeld, Spheres in infinite-dimensional normed spaces are Lipschitz contractible, Proc. Amer. Math. Soc. 88 (1983), no. 3, 439-445.

[2] K. Bolibok, Construction of a Lipschitzian retraction in the space $c_{0}$, Ann. Univ. Mariae Curie-Skłodowska Sect. A 51 (1997), no. 2, 43-46.

[3] - Minimal displacement and retraction problems in the space $l^{1}$, Nonlinear Anal. Forum 3 (1998), 13-23.

[4] K. Bolibok and K. Goebel, A note on minimal displacement and retraction problems, J. Math. Anal. Appl. 206 (1997), no. 1, 308-314.

[5] C. Franchetti, Lipschitz maps and the geometry of the unit ball in normed spaces, Arch. Math. (Basel) 46 (1986), no. 1, 76-84.

[6] K. Goebel, A way to retract balls onto spheres, J. Nonlinear Convex Anal. 2 (2001), no. $1,47-51$.

[7] K. Goebel and W. A. Kirk, Topics in Metric Fixed Point Theory, Cambridge Studies in Advanced Mathematics, vol. 28, Cambridge University Press, Cambridge, 1990.

[8] K. Goebel and T. Komorowski, Retracting balls onto spheres and minimal displacement problem, Fixed Point Theory and Applications (Marseille, 1989), Pitman Res. Notes Math. Ser., vol. 252, Longman Scientific \& Technical, Harlow, 1991, pp. 155-172.

[9] T. Komorowski and J. Wośko, A remark on the retracting of a ball onto a sphere in an infinite-dimensional Hilbert space, Math. Scand. 67 (1990), no. 2, 223-226.

[10] P. K. Lin and Y. Sternfeld, Convex sets with the Lipschitz fixed point property are compact, Proc. Amer. Math. Soc. 93 (1985), no. 4, 633-639.

[11] B. Nowak, On the Lipschitzian retraction of the unit ball in infinite-dimensional Banach spaces onto its boundary, Bull. Acad. Polon. Sci. Sér. Sci. Math. 27 (1979), no. 11$12,861-864$.

Kazimierz Goebel: Instytut Matematyki, Universytet M. Curie-Skłodowskiej (UMCS), 20-031 Lublin, Poland

E-mail address: goebel@golem.umcs.lublin.pl 


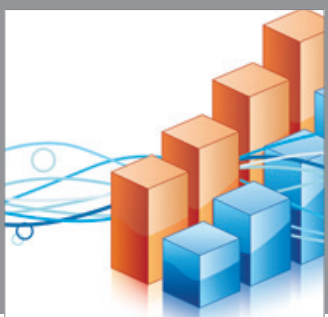

Advances in

Operations Research

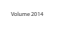

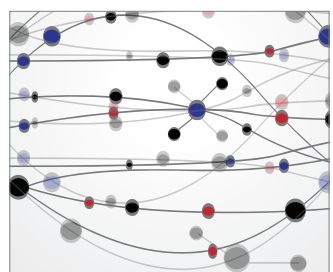

\section{The Scientific} World Journal
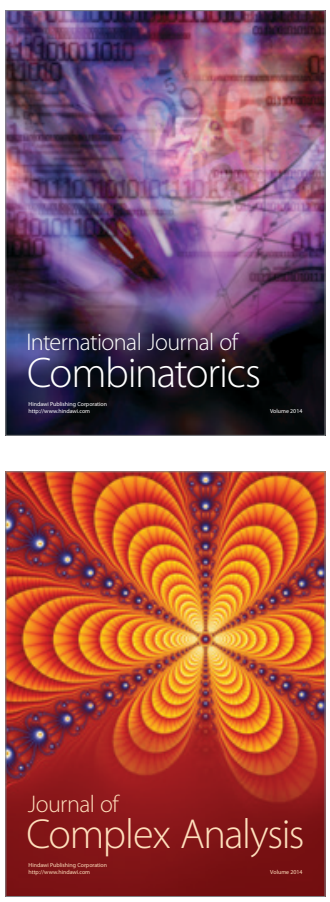

International Journal of

Mathematics and

Mathematical

Sciences
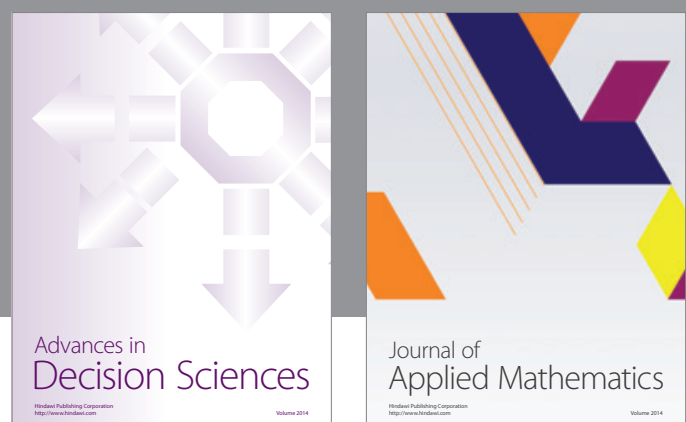

Journal of

Applied Mathematics
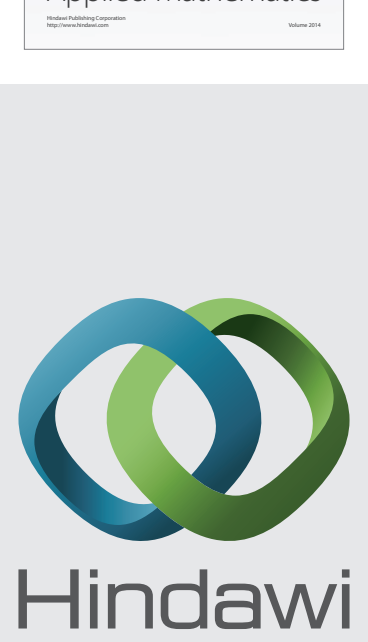

Submit your manuscripts at http://www.hindawi.com
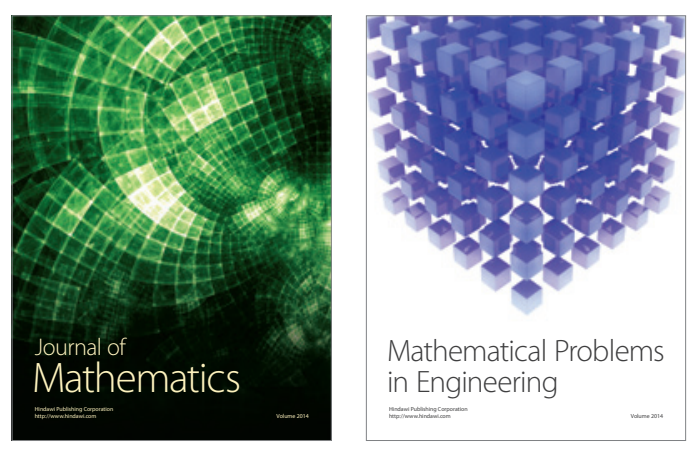

Mathematical Problems in Engineering
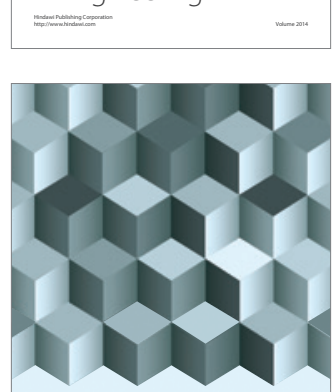

Journal of

Function Spaces
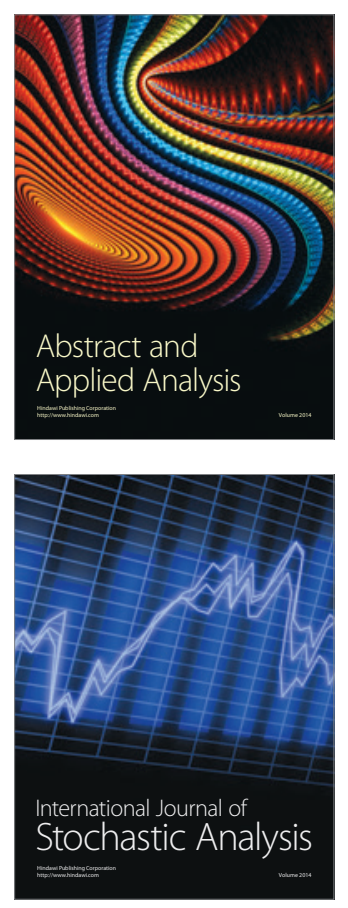

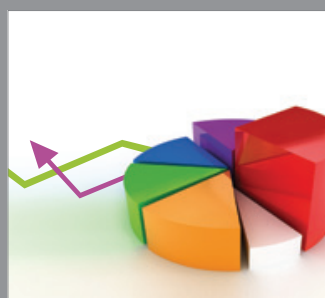

ournal of

Probability and Statistics

Promensencen
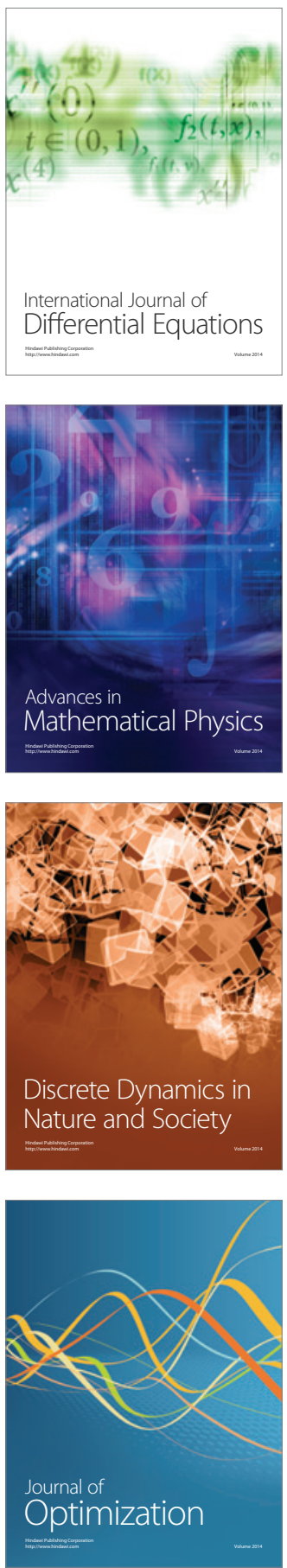